\title{
AUDIOVISUAL SPEAKER DIARIZATION OF TV SERIES
}

\author{
Xavier Bost, Georges Linarès, Serigne Gueye* \\ LIA, University of Avignon, 339 chemin des Meinajariès, 84000 Avignon, France
}

\begin{abstract}
Speaker diarization, also known as the "who spoke when?" task, may be difficult to achieve when applied to narrative films, where speakers usually talk in adverse acoustic conditions: background music, sound effects, wide variations in intonation may hide the inter-speaker variability and make audio-based speaker diarization approaches error prone. On the other hand, such fictional movies exhibit strong regularities at the image level, particularly within dialogue scenes. In this paper, we propose to perform speaker diarization within the dialogue scenes of TV series by combining the audio and video modalities: speaker diarization is first performed by using each of these modalities; the two resulting partitions of the instance set are then optimally matched, before the remaining instances, corresponding to cases of disagreement between the modalities, are finally processed. The results obtained by applying such a multi-modal approach to fictional films turn out to outperform those obtained by using a single modality.
\end{abstract}

Index Terms - Speaker diarization, multi-modal fusion, video structuration

Cite as:

X. Bost, G. Linarès, S. Gueye.

Audiovisual speaker diarization of TV series.

2015 IEEE International Conference on Acoustics, Speech and Signal Processing (ICASSP).

doi: 10.1109/ICASSP.2015.7178882

\section{INTRODUCTION}

Speaker diarization (SD) consists in assigning the spoken segments of an audio stream to their respective speakers, without any prior knowledge about the speakers involved nor their number. Most of state of the art systems rely on a two-step approach, performing first speaker turn detection followed by single-speaker segment clustering. This last stage is usually based on hierarchical clustering ([1]) and, more recently, mathematical programming ([2, 3]).

SD was first applied to audio-only streams produced in adverse, but controlled, conditions, such as telephone conversations, broadcast news, meetings... More recently, SD was extended to video streams, facing the critical issue of processing contents produced in an uncontrolled and variable environment.

In [4], the authors apply standard speaker diarization tools to the audio channel of various video documents collected on the web, resulting in high Diarization Error Rates (DER) in comparison to the scores obtained with the usual audio streams.

Recent works intend to perform speaker diarization of such audiovisual contents by using jointly the multiple sources of informa-

* This work was partially supported by the French National Research Agency (ANR) CONTNOMINA project (ANR-07-240) and the Research Federation Agorantic, Avignon University. tion conveyed by multimedia streams. The multi-modal speaker diarization method introduced in [5] relies on an early combination of audio and video GMMs, before applying a standard BIC-based agglomerative algorithm to the resulting features. This technique is evaluated on the AMI corpus which gathers audiovisual recordings of four speakers playing roles in a meeting scenario.

In [6], the authors make use of both face clustering and speaker diarization to perform face identification in TV debates: face clustering and speaker diarization are first processed independently. Then, the current speaker is identified by selecting the best modality. Finally, local information about the current speaker identity are propagated to the whole cluster of the corresponding utterance.

In [3], the authors make use of an intermediate fusion approach to guide speaker diarization in TV broadcast by adding to the set of speech turns new instances originating in other sources of information: the names written on the screen when a guest on a reporter is introduced as well as the corresponding identities. Adding such instances allows to constrain the clustering process leading to purer classes of speakers.

Finally, audio-based SD has already been applied ([7]) to TV series, but as a mean among other modalities to structure its contents.

In this paper, we propose to use the visual structure of narrative movies to perform audiovisual speaker diarization within each dialogue scene of TV series episodes.

Diarization on such movies presents some specific difficulties due to audio-video asynchrony that limit the performance of videoonly based SD systems: the current speaker may not be filmed, the camera focusing on the reaction of the character he is talking to. These highly unpredictable asynchrony issues make necessary the joint use of audio and video features.

On the other hand, movie dialogue scenes exhibit formal regularities at a visual level, with two alternating and recurring shots, each one corresponding to one of the two speakers involved. Once automatically detected, such patterns could limit the interactivity scheme in which diarization is performed.

This paper focus on speaker diarization in TV series. We propose to perform independently audio and video-based speaker diarization, before merging the resulting partitions of the spoken segments in an optimal way. The two modalities are expected to be uncorrelated in their respective mistakes, and to compensate each other.

The way dialogue scenes are visually detected is described in Section 2; the method used to perform mono-modal speaker diarization is described in section 3 and the way the two resulting partitions of the utterance set are combined is described in section 4. Experimental results are given and discussed in section 5 .

\section{DIALOGUE SCENES VISUAL DETECTION}

Relying on specific shot patterns, the detection of the dialogue scenes requires the whole video stream be split into shots, before these ones are compared and labelled according their similarities. 


\subsection{Shot cut and shot similarity detection}

The whole video stream can be regarded as a sequence of fixed images (or frames) displayed on the screen at a constant rate able to simulate for human eyes the continuity of motion. Moreover, a video shot, as stated in [8], is defined as an "unbroken sequence of frames taken from one camera". A new shot can then be detected by comparing the current image to the next one: a substantial difference between two temporally adjacent images is indicative of a shot cut. Conversely, the current shot is considered as similar to a past one if the first image of the former substantially looks like the last one of the latter.

Both tasks, shot cut detection as well as shot similarity detection, rely on image comparison. For this comparison purpose, images are described by using 3-dimension histograms of the image pixel values in the HSV color space. Comparison between images is then performed by evaluating the correlation between the corresponding color histograms. Nonetheless, different images may share the same global color histogram, resulting in a irrelevant similarity: information about the spatial distribution of the colors on the image is then reintroduced by splitting the image into 30 blocks, each associated to its own color histogram; block-based comparison between image is then processed as described in [8].

The two correlation thresholds needed to perform both tasks, shot cut detection as well as shot similarity detection, are estimated by experiments on a development set of TV series episodes (see Subsection 5.2).

\subsection{Dialogue visual patterns extraction}

Once shots are extracted and similar ones are detected, shot patterns typical of short dialogue scenes can be detected. Let $\Sigma=$ $\left\{l_{1}, \ldots, l_{m}\right\}$ be a set of possible shot labels, two shots sharing the same label if they are hypothesized as similar.

The following regular expression $r\left(l_{1}, l_{2}\right)$ corresponds to a subset of all the possible shot sequences $\Sigma^{*}=\bigcup_{n \geqslant 0} \Sigma^{n}$ :

$$
r\left(l_{1}, l_{2}\right)=\Sigma^{*} l_{1}\left(l_{2} l_{1}\right)^{+} \Sigma^{*}
$$

The set $\mathcal{L}\left(r\left(l_{1}, l_{2}\right)\right.$ of sequences captured by the regular expression 1 corresponds to shot label sequences containing an occurrence of $l_{2}$ inserted between two occurrences of $l_{1}$, with a possible repetition of the alternation $\left(l_{2}, l_{1}\right)$, whatever be the previous and following shot labels. Such a regular expression formalizes the "twoalternating-and-recurring-shots" pattern mentioned in Section 1 as typical of dialogue scenes involving two characters.

Figure 1 shows an example of a shot sequence matching the regular expression 1.

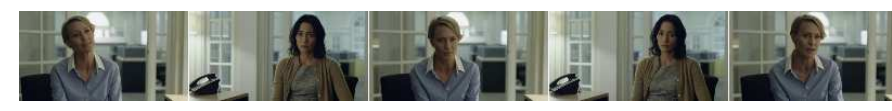

Fig. 1. Example of shot sequence $\ldots l_{1} l_{2} l_{1} l_{2} l_{1} \ldots$ captured by the regular expression 1 for two shot labels $l_{1}$ and $l_{2}$.

A movie can be described as a finite sequence $\mathbf{s}=s_{1} \ldots s_{k}$ of $k$ shot labels, with $s_{i} \in \Sigma$. The set of patterns $\mathcal{P}(\mathbf{s}) \subseteq \Sigma^{2}$ associated with the movie shot sequence $\mathbf{s}$ can be defined as follows:

$$
\mathcal{P}(\mathbf{s})=\left\{\left(l_{1}, l_{2}\right) \in \Sigma^{2} \mid \mathbf{s} \in r\left(l_{1}, l_{2}\right)\right\}
$$

The set of patterns $\mathcal{P}(\mathbf{s})$ contains all the pairs of shots alternating with each other according to the rule 1 .

In order to increase the speech coverage of such visual patterns, isolated expressions of the alternating shot pairs involved in a pattern are also taken into account.

The scenes of our corpus movies that match the regular expression 1 appear to contain relatively few speech (13.15 seconds in average) but cover more than half $(53.10 \%)$ of the total amount of speech of a movie. Not surprisingly, the number of speakers involved in such scenes with two alternating shots is close to two speakers (1.84), with a standard deviation of 0.57 : the scenes with only one speaker are mainly the shortest ones, where the probability that one of the two speakers remains silent increases.

\section{MONO-MODAL SPEAKER DIARIZATION}

Widely available, the movie subtitles are here used to approximate utterance boundaries. As an exact transcription of each utterance, they usually match it temporally, despite some slight and unpredictable latency before they are displayed and after they disappear. When such a latency was too high, the utterance boundaries were manually adjusted. Moreover, each subtitle is generally associated with a single speaker, and in the remaining cases where two speakers are involved in a single subtitle, speech turns are indicated, allowing to split the whole subtitle into the two corresponding utterances.

\subsection{Audio and visual features}

Once delimited, utterances can be described using either acoustic or visual features.

The acoustic parameterization of utterances relies, as a state of the art technique used in the speaker verification field, on the i-vectors model ([9]). After 19 cepstral coefficients plus energy are extracted, a 64-components GMM/UBM is trained on the whole corpus (described in Subsection 5.1); the total variability matrix is then trained on all the spoken segments of the currently processed movie, and 20-dimension i-vectors are finally extracted, each associated with a single utterance. I-vectors are extracted using the ALIZE toolkit described in [10].

On the other hand, the visual parameterization of an utterance relies on its temporal distribution over the shots, as labelled according their similarities (see subsection 2.1).

Considering the set $\Sigma=\left\{l_{1}, \ldots, l_{m}\right\}$ of shot labels involved in a movie, the $i$-th dimension of $\mathbb{R}_{+}^{m}$ is associated to the $i$-th shot label. Each utterance $\mathbf{u}=\left(u_{0}, \ldots, u_{m}\right)$ is then described as an $m$-dimension vector, where the $i$-th component $u_{i}$ corresponds to the overlapping time in seconds between the utterance $\mathbf{u}$ and the shot label $l_{i}$.

Figure 2 shows the distribution over time of the two alternating shots of Figure 1, here labelled $\left(c_{126}, c_{127}\right)$. The top line reports the alternation of both shots over time; the bottom line contains the

\begin{tabular}{|c|c|c|c|c|c|c|}
\hline C126 & & C127 & & C126 & C127 & C126 \\
\hline 202 & 203 & 204 & 205 & 206 & 207 & 208 \\
\hline
\end{tabular}
seven utterances covered by the sequence.

Fig. 2. Shot sequence .... $c_{126} c_{127} c_{126} c_{127} c_{126} \ldots$ for two shot labels $c_{126}$ and $c_{127}$ (top line) with the covered utterances (bottom line). 
For instance, The utterance $\mathbf{u}^{(\mathbf{2 0 5})}$ overlaps the two shots and will then be set to 1.56 (seconds) for its 126 -th component, to 1.16 for its 127 -th component, and to zero for all the other ones.

\subsection{P-median clustering}

The $n$ utterances covered by a particular pattern can then be described either according audio-only features, resulting in a set $\mathcal{U}_{a}$ of $n$ 20-dimension i-vectors or by using visual-only features, resulting in a set $\mathcal{U}_{v}$ of $n m$-dimension vectors, where $m$ denotes the number of shot labels in the movie.

Both sets $\mathcal{U}_{a}$ and $\mathcal{U}_{v}$ are first partitioned into two clusters each, the average number of speakers by pattern (1.84), as well as its standard variation, allowing such an a priori assumption.

With such a fixed number of clusters, the partition problem can be modelled using the $p$-median problem. The $p$-median problem ([11, 12]) belongs to the family of facility location problems: $p$ facilities must be located among possible candidate sites such that the total distance between demand nodes and the nearest facility is minimized.

The $p$-median problem can be transposed into the cluster analysis context ([13]) with a predefined number of classes. The instances to cluster into $p$ classes correspond to the demand nodes and each instance may be chosen as one of the $p$ class centers. Choosing the centers so as to minimize the total distance between the instances and their nearest center results in compact classes with medoid centers.

Considering the set $\mathcal{U}$ of $n$ utterances covered by a pattern, the clustering problem can be modelled using the following binary decision variables: $x_{i}=1$ if the $i$-th utterance $u^{(i)}$ is selected as one of the $p$ cluster centers, $x_{i}=0$ otherwise; $y_{i j}=1$ if $u^{(i)}$ is assigned to the cluster center $u^{(j)}, y_{i j}=0$ otherwise. The model constants are the number of centers $p$ as well as the distance coefficients $d_{i j}$ between the utterances $u^{(i)}$ and $u^{(j)}$. The distance metric is the euclidean one in the case of the video-based utterance vectors and the normalized euclidean distance in the case of audio-based utterance i-vectors.

The $p$-median clustering problem can then be modelled as the following integer linear program, closely related to the program described in $[2,14]$ :

$$
\text { (P1) }\left\{\begin{array}{l}
\min \left(\sum_{i=1}^{n} \sum_{j=1}^{n} d_{i j} y_{i j}\right) \\
\text { s.t. } \mid \begin{array}{l}
\sum_{j=1}^{n} y_{i j}=1 \\
\sum_{i=1}^{n} x_{i}=p \\
y_{i j} \leqslant x_{i} \\
x_{i} \in\{0,1\} \\
y_{i j} \in\{0,1\} \quad i=1, \ldots, n
\end{array} \\
\end{array}\right.
$$

The first constraints $\sum_{j=1}^{n} y_{i j}=1$ ensures that each utterance is assigned to exactly one center; the second one $\sum_{i=1}^{n} x_{i}=p$ that exactly $p$ centers are chosen; the third ones $y_{i j} \leqslant x_{i}$ prevent an utterance from being assigned to a non-center one.

Setting $p:=2$ and solving twice the integer linear program (P1), once for the utterance set $\mathcal{U}_{a}$ described by audio features, and then for the utterance set $\mathcal{U}_{v}$ relying on visual features, results in two distinct bipartitions of the same utterance set.

\section{MULTI-MODAL COMBINATION}

\subsection{Optimal matching fusion}

The two bipartitions of the utterance set are then merged by solving the classical maximum weighted matching in a bipartite graph.

On Figure 3, the set of utterances $\mathcal{U}=\left\{\mathbf{u}^{(\mathbf{1})}, \mathbf{u}^{(\mathbf{2})}, \mathbf{u}^{(\mathbf{3})}, \mathbf{u}^{(\mathbf{4})}\right\}$ is twice partitioned using the audio and video modalities, resulting in two different partitions $\mathcal{Q}_{a}=\left\{Q_{a}^{(1)}, Q_{a}^{(2)}\right\}$ and $\mathcal{Q}_{v}=$ $\left\{Q_{v}^{(1)}, Q_{v}^{(2)}\right\}$.

A bipartite weighted graph $\mathcal{G}=\left(\mathcal{Q}_{a}, \mathcal{Q}_{v}, \mathcal{E}\right)$, where $\mathcal{E}=\mathcal{Q}_{a} \times$ $\mathcal{Q}_{v}$, can then be defined by assigning to each edge $\left(Q_{a}^{(i)}, Q_{v}^{(j)}\right) \in \mathcal{E}$ a weight $w_{i j}$ corresponding to the sum of the duration of the utterances that the sets $Q_{a}^{(i)}$ and $Q_{v}^{(j)}$ have in common.

The edges of the bipartite graph on Figure 3 are thus weighted by assuming a same duration of 1 for all the utterances $\mathbf{u}^{(\mathbf{1})}, \ldots, \mathbf{u}^{(\mathbf{4})}$.

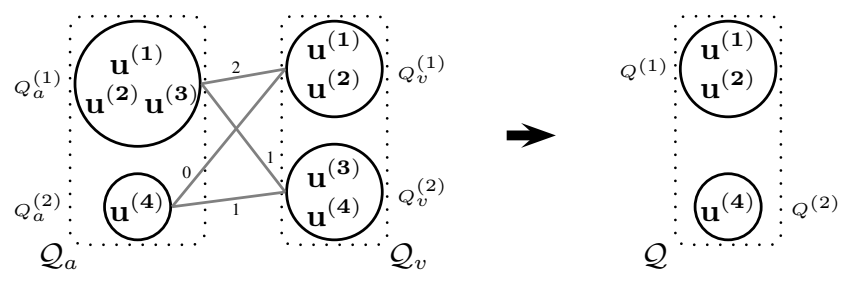

Fig. 3. Set partitions fusion using maximum weighted matching in a bipartite graph

The best matching between both partitions consists in choosing non-adjacent edges (without any node in common) so that the sum of their weights is maximized. By using a decision variable $y_{i j}$ such that $y_{i j}=1$ if the edge $\left(Q_{a}^{(i)}, Q_{v}^{(j)}\right)$ is chosen, $y_{i j}=0$ otherwise, the problem can be modelled as follows for two bipartitions:

$$
\text { (P2) }\left\{\begin{array}{l|l}
\max \left(\sum_{i=1}^{2} \sum_{j=1}^{2} w_{i j} y_{i j}\right) & \\
\text { s.t. } & \begin{array}{l}
\sum_{j=1}^{2} y_{i j} \leqslant 1 \\
\sum_{i=1}^{2} y_{i j} \leqslant 1 \\
y_{i j} \in\{0,1\}
\end{array} \quad(i, j) \in\{1,2\}^{2}
\end{array}\right.
$$

The first and second constraints ensure that only non adjacent edges will possibly be chosen.

In the example of Figure 3, the best choice consists in assigning $Q_{a}^{(1)}$ to $Q_{v}^{(1)}$ and $Q_{a}^{(2)}$ to $Q_{v}^{(2)}$, for a total cost of 3 .

Once the matching choice is made by solving the problem $(\mathrm{P} 2)$, the matching subsets are intersected, resulting in a new set $\mathcal{Q}$ of subsets of $\mathcal{U}$ corresponding to cases of agreement between the two modalities: the subsets obtained are expected to contain segments both acoustically close to each other and uttered as the corresponding speaker is filmed. Conversely, the residual segments $\left(\mathbf{u}^{(3)}\right.$ in the example of Figure 3) are discarded as cases of disagreement between the audio and visual modalities, either because the utterance is acoustically atypical or because of asynchrony between the utterance and the character currently filmed. 


\subsection{Reallocation of discarded utterances}

The residual utterances are finally reallocated to the closest medoids of the refined clusters resulting from the combination of the audio and visual modalities.

This stage of reallocation relies on the audio-only features of the remaining utterances: possibly discarded because of their visual asynchrony, such utterances might not be correctly reallocated by relying on the visual modality. On the other hand, using the audio modality to achieve such a reallocation is expected to be more robust than when performing the audio-only clustering described in subsection 3.2: by using medoids of clusters refined by the use of the video modality, some errors made during the audio-only stage are expected to be here avoided. Moreover, medoid, being less sensitive to outliers than centroid, is expected to properly handle the case of impure clusters containing isolated misclassified utterances resulting from a joint error of both modalities.

\section{EXPERIMENTS AND RESULTS}

\subsection{Corpus}

For experimental purpose, we used the first seasons of three TV series: Breaking Bad (abbreviated bb), Game of Thrones (got), and House of Cards (hoc). We manually annotated three episodes of each series by indicating shot cuts, similar shots, speech segments as well as the corresponding speakers. The total amount of speech in these nine episodes represents a bit more than three hours (3:12).

\subsection{Shot cuts and shot similarities detection}

The evaluation of shot cut detection relies on a classical F1-score ([15]). For the shot similarity detection task, an analogous F1-score is used: for each shot, the list of shots hypothesized as similar to the current one is compared to the reference list of similar shots; if both lists intersect in a non-empty set, the shot is considered as correctly paired with its list. As both these image processing tasks require thresholds estimation, a development subset of six episodes is here used. Average results on DEV and TEST sets are reported in Table 1.

Table 1. Average results obtained for shot cut and shot similarity detection

\begin{tabular}{|c|c|ccc|}
\hline & shot cut & \multicolumn{3}{|c|}{ shot similarity } \\
\cline { 2 - 5 } & F1-score & precision & recall & F1-score \\
\hline avg. DEV & $\mathbf{0 . 9 7}$ & 0.90 & 0.88 & $\mathbf{0 . 8 9}$ \\
\hline \hline avg. TEST & $\mathbf{0 . 9 9}$ & 0.91 & 0.90 & $\mathbf{0 . 9 0}$ \\
\hline
\end{tabular}

The results obtained for the shot similarity detection task (F1score amounting to 0.90 ) are expected to make reliable the visual detection of alternating, recurring shots as typical of short dialogue scenes involving two characters.

\subsection{Speaker diarization within dialogue scenes}

Speaker diarization, performed within each dialogue scene as hypothesized from visual clues, is evaluated using the single show DER ([16]): the DER is computed for each dialogue scene before the results are averaged according each dialogue duration. Results are given for the mono-modal speaker diarization step for both modalities, audio and video. The optimal matching (denoted om) performed during the multi-modal fusion step is evaluated in two ways: first by discarding from scoring the utterances for which the two modalities disagree (denoted om-ra). In this case, the resulting speech coverage of the scored utterances in indicated in percentage in parenthesis. Moreover, results are also given when the optimal matching between both modalities is followed by a step of audio reallocation of the remaining utterances (denoted $o m+r a$ ). For the sake of comparison, the results obtained by optimizing jointly in a weighted sum (denoted $w s$ ) the two $p$-median mono-modal objective functions are also reported. Finally, an oracle score is estimated by labelling the utterances according the reference speaker when at least one of both modalities succeeds in retrieving it.

Table 2. Single show Diarization Error Rate obtained for all episodes

\begin{tabular}{|c|c|c|c|c|c|c|}
\hline & \multicolumn{2}{|c|}{ mono-modal } & \multirow[t]{2}{*}{ oracle } & \multicolumn{3}{|c|}{ multi-modal } \\
\hline & audio & video & & om-ra & $o m+r a$ & $w s$ \\
\hline$\overline{c b b-1}$ & $\overline{25.2}$ & 26.9 & $\overline{8.3}$ & $1818.0_{(67.7)}$ & $\overline{24.0}$ & 26.9 \\
\hline$b b-2$ & 26.6 & 24.5 & 8.2 & $17.2_{(69.7)}$ & 20.4 & 24.5 \\
\hline$b b-3$ & 26.8 & 26.9 & 9.6 & $17.1_{(67.4)}$ & 24.7 & 27.3 \\
\hline got-1 & 22.6 & 24.7 & 7.6 & $13.1_{(69.2)}$ & 21.1 & 24.5 \\
\hline got -2 & 28.7 & 27.7 & 10.2 & $20.0_{(68.2)}$ & 25.9 & 27.0 \\
\hline got -3 & 12.8 & 29.4 & 5.3 & $9.99_{(71.1)}$ & 13.1 & 28.2 \\
\hline hoc-1 & 17.5 & 21.9 & 3.8 & $10.0_{\text {(71.6) }}$ & 17.7 & 22.2 \\
\hline hoc -2 & 21.4 & 29.4 & 10.2 & 15.4 (70.6) & 20.8 & 29.4 \\
\hline hoc -3 & 20.6 & 25.6 & 6.9 & $12.8_{(70.2)}$ & 20.6 & 25.4 \\
\hline$\overline{\text { avg. }}$. & $\overline{\overline{22.5}}$ & $\overline{26.3}$ & $\overline{\overline{7.8}}$ & $\overline{\overline{14.8}}$ & $\overline{20.9}$ & $\overline{26.2}$ \\
\hline
\end{tabular}

As can be seen, the results obtained by performing mono-modal speaker diarization are in average slightly better for the audio modality than for the video one. Nonetheless, the computed oracle shows that both modalities are not redundant: by managing to combine them perfectly, the DER would decrease dramatically (from $22.5 \%$ to $7.8 \%$ for the audio modality, and from $26.3 \%$ to $7.8 \%$ for the video one), which confirms that both these modalities are highly complementary for the speaker diarization task and that the errors made are not correlated.

Moreover, when both modalities are combined, resulting in a new partial clustering of the utterance set, the DER remains relatively low if about $30 \%$ of the utterances, corresponding of cases of disagreement between both modalities, are discarded from the evaluation (DER amounting to $14.8 \%$ for $69.5 \%$ of speech covered).

Not surprisingly, while processing the critical $30 \%$ remaining utterances, the DER tends to increase (from $14.8 \%$ to $20.9 \%$ ) but is still lower than the DER obtained for the single audio modality $(22.5 \%)$, a relative improvement of $7.11 \%$.

\section{CONCLUSION}

In this paper, we proposed to perform audiovisual speaker diarization within short scenes of TV series visually hypothesized as dialogues between two characters. Speaker diarization is first performed separately for audio and visual features of the utterances by using the $p$-median model, before both resulting bipartitions of the utterance set are optimally matched in new clusters corresponding to cases of agreement between both modalities. The isolated remaining utterances for which both modalities disagree are then acoustically assigned to the closest centroids of the newly created clusters, expected to be more robust than when based on an audio-only approach. The experimental results obtained by using both modalities turn out to outperform those obtained by purely mono-modal approaches. 


\section{REFERENCES}

[1] Nicholas Evans, Simon Bozonnet, Dong Wang, Corinne Fredouille, and Raphaël Troncy, "A comparative study of bottomup and top-down approaches to speaker diarization," Audio, Speech, and Language Processing, IEEE Transactions on, vol. 20, no. 2, pp. 382-392, 2012. 1

[2] Grégor Dupuy, Mickael Rouvier, Sylvain Meignier, and Yannick Estève, "I-vectors and ilp clustering adapted to crossshow speaker diarization.," in INTERSPEECH, 2012. 1, 3

[3] Hervé Bredin, Johann Poignant, et al., "Integer linear programming for speaker diarization and cross-modal identification in tv broadcast," in the 14rd Annual Conference of the International Speech Communication Association, INTERSPEECH, 2013. 1

[4] Pierre Clément, Thierry Bazillon, and Corinne Fredouille, "Speaker diarization of heterogeneous web video files: A preliminary study," in Acoustics, Speech and Signal Processing (ICASSP), 2011 IEEE International Conference on. IEEE, 2011, pp. 4432-4435. 1

[5] G. Friedland, H. Hung, and Chuohao Yeo, "Multi-modal speaker diarization of real-world meetings using compresseddomain video features," in Acoustics, Speech and Signal Processing, 2009. ICASSP 2009. IEEE International Conference on, April 2009, pp. 4069-4072. 1

[6] Meriem Bendris, Benoit Favre, Delphine Charlet, Géraldine Damnati, Gregory Senay, Rémi Auguste, and Jean Martinet, "Unsupervised face identification in tv content using audio-visual sources," in Content-Based Multimedia Indexing (CBMI), 2013 11th International Workshop on. IEEE, 2013, pp. 243-249. 1

[7] Hervé Bredin, "Segmentation of tv shows into scenes using speaker diarization and speech recognition," in Acoustics, Speech and Signal Processing (ICASSP), 2012 IEEE International Conference on. IEEE, 2012, pp. 2377-2380. 1

[8] Irena Koprinska and Sergio Carrato, "Temporal video segmentation: A survey," Signal processing: Image communication, vol. 16, no. 5, pp. 477-500, 2001. 2

[9] Najim Dehak, Patrick Kenny, Réda Dehak, Pierre Dumouchel, and Pierre Ouellet, "Front-end factor analysis for speaker verification," Audio, Speech, and Language Processing, IEEE Transactions on, vol. 19, no. 4, pp. 788-798, 2011. 2

[10] Jean-François Bonastre, Frédéric Wils, and Sylvain Meignier, "Alize, a free toolkit for speaker recognition.," in $\operatorname{ICASSP}(1)$, 2005, pp. 737-740. 2

[11] S Louis Hakimi, "Optimum locations of switching centers and the absolute centers and medians of a graph," Operations research, vol. 12, no. 3, pp. 450-459, 1964. 3

[12] SL Hakimi, "Optimum distribution of switching centers in a communication network and some related graph theoretic problems," Operations Research, vol. 13, no. 3, pp. 462-475, 1965. 3

[13] Ted D Klastorin, "The p-median problem for cluster analysis: A comparative test using the mixture model approach," Management Science, vol. 31, no. 1, pp. 84-95, 1985. 3

[14] Grégor Dupuy, Sylvain Meignier, Paul Deléglise, and Yannick Esteve, "Recent improvements on ilp-based clustering for broadcast news speaker diarization," in Proceedings of Odyssey, 2014. 3
[15] John S Boreczky and Lawrence A Rowe, "Comparison of video shot boundary detection techniques," Journal of Electronic Imaging, vol. 5, no. 2, pp. 122-128, 1996. 4

[16] Mickael Rouvier, Grégor Dupuy, Paul Gay, Elie Khoury, Teva Merlin, and Sylvain Meignier, "An open-source state-of-the-art toolbox for broadcast news diarization," in INTERSPEECH, 2013, number EPFL-CONF-192762. 4 